\title{
Segmentation of Food Images using Morphological Operations with Watershed Segmentation Technique
}

\author{
Shital V. Chavan \\ Department of Computer Engineering \\ Pimpri Chinchwad College of Engineering \\ Pune-44
}

\author{
S. S. Sambare \\ Department of Computer Engineering \\ Pimpri Chinchwad College of Engineering
}

Pune-44

\begin{abstract}
Person across the world are now becoming more conscious about their health and weight. Accuracy of the calorie measuring system is depending on food image analysis. The proper analysis of an image is based on image segmentation technique and is one of the important steps in image analysis. Multiple image segmentation techniques exist for extracting requires objects from an image. Different image segmentation techniques based on edge detection, region growing, based on clustering and thresholding are applied on food images but these techniques are not useful for segmenting the overlapped regions of food. Watershed segmentation technique is used to solve the problem of over segmentation and segmenting small regions which are overlapped to each other but this segmentation results are not more accurate for increasing accuracy of segmentation result. In this paper work hybrid model is proposed by combining Morphological Operations and Watershed Segmentation technique (MOWS) to segment overlapped regions of food with their shape. Watershed Segmentation and MOWS are implemented in MATLAB and Analysis is done based on their PSNR values. Analysis shows that proposed hybrid model MOWS is given more accurate result than watershed segmentation.
\end{abstract}

\section{Keywords}

Image Segmentation, Edge Detection, Watershed Segmentation Technique, Morphological Operations.

\section{INTRODUCTION}

Every individual having their own eating habit, some having good healthy eating habit or some having unhealthy eating habit. Which is directly affected on their body Because of unhealthy eating habits person gives open invitation for any disease. So calorie measurement system will tell user how much he or she has to eat their food daily as per their lifestyle or by taking images of their food before and after eat. The calorie measuring system is also depending on food image analysis and the proper analysis of an image is based on image segmentation technique which separate different regions and helps to measure quantity of food user taken. Normally food images are used in calorie measurement systems to calculate how much calorie user eaten, by analyzing food image before and after eating. From normal human eye vision nobody accurately say how much calorie is getting from food. So there is an urgent need of control and monitoring system required to control on dietary intake. Computer vision technology, which helps in processing the

any type of images and extracting meaningful information from images. Images are considered as one of the most important medium to carry information about content of images which are present in images [1]. In image analysis, image segmentation is one of the most important step for processing images and good segmentation results into accurate results. Image segmentation is the process of dividing the digital image into multiple segments, i.e. set of pixel and pixels in a region are similar according to some similarity criteria such as color, intensity or texture, to identify objects and boundaries present in an image [2].Image segmentation techniques are mainly dividing into four categories as shown in figure 1.

Thresholding methods convert color image into gray level image and separate out area of interest from background. In thresholding 1 represent foreground image and 0 represent background image. In Edge Detection method edges are extracted and different regions are separate out. In clustering method basically k-means clustering is used and which cluster the pixels which are similar to each other into same cluster. Clustering is unsupervised technique. In region based segmentation image is partitions into different regions based on predefined condition or seed point.

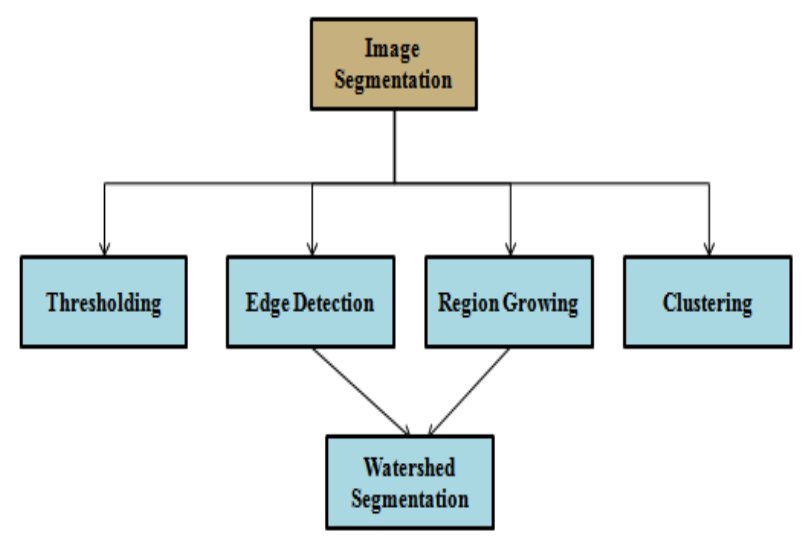

Figure 1: Image Segmentation Techniques

For segmenting overlapped regions watershed segmentation technique is used and it is also similar to region based segmentation method. Watershed segmentation algorithm is a based on mathematical morphology. For binary image analysis it is mainly used. Watershed algorithm is based on the flooding process implementation and that is the foundation of $2 \mathrm{D}$ plus the height of $3 \mathrm{D}$ foundation. There is some local minimum point in the image. If a hole is played in each tiny point, water will slowly go into the surface from these holes, starting from the lowest minimum point, then from the lowest point water inundated water basin. a dam is built when water surface from two different minimum points are gather together. At the end of the process, each minimum point is surrounded by corresponding water basin of the dam, the set of whole dam forms a watershed. Different water basin is made up different region of the image, and the image segmentation is realized [3]. 


\section{RELATED WORK}

SUN Hui-jie et al [3]. In this paper proposed an improved watershed image segmentation algorithm to solve the problems of noise sensitivity and over segmentation. The proposed algorithm is combined by region growing with classical watershed algorithm. Shannon entropy is used as a parameter for region growing. The particle swarm optimization algorithm is used to search global optimization of the objective function which is constructed by using region growing and classical watershed segmentation. The Experimental results shows that the new watershed image segmentation algorithm solve the problem of over segmentation and turns out to be an efficient, accurate algorithm.

Xuemei Cui, Guowei Yang, Yan Deng and Shaolong Wu et al [4]. In this paper, proposed a new segmentation algorithm which is base on improved watershed algorithm. With watershed segmentation by marking on the different foreground and background objects, can obtain better effect of segmentation. Result of watershed segmentation is based on gradient image and by applying opening and closing function on image gradient image is obtained. From the new algorithm new tag extraction method is designed, by using the method of opening and closing by reconstruction operators to obtain marks and by controlling the closed reconstruction of structural elements in order to interest in the gradient image area the size of the tag. Then, extracted tag is used as function for segmenting the region. Lastly image is segmented by the gradient of the image which is modified in this paper work.

Md. Habibur Rahman and Md. Rafiqul Islam et al [5]. In this paper, proposed a method to overcome the problem of over segmentation by using an adaptive thresholding and masking mechanism with watershed algorithm. The proposed modified watershed algorithm for image segmentation overcome over segmentation problem, each color channel is separated and on that adaptive thresholding and masking mechanism is applied. before combining the segmentation from each channel into the final one. The experimental results are obtained using metrics such as PSNR, MSE, PSNRRGB and Color Image Quality Measure (CQM) based on reversible YUV color transformation. The proposed modified watershed approach improves the image segmentation performance and the proposed MWS method is faster than other segmentation algorithms.

Parisa Pouladzadeh, Shervin Shirmohammadi and Rana AlMaghreb et al [6]. In this paper, proposed a food calorie and nutrition measurement system that can help dietitians and patients to measure and manage their daily food intake. The system uses nutritional fact tables and food images. System uses the built-in camera of such mobile devices and records a photo of the food before and after eating to measure the how much of calorie and nutrient components user have eaten. Edge detection method is used to separate out different regions present in image and after that SVM classification technique is used to classify extracted regions. From that calorie measurement is done, but edge detection method not work well for image which having fruit salad or food portions which are mixed.

Nandhini. P, Dr. J. Jaya et at [7]. This paper uses various image processing steps to provides an efficient method for identifying the defected parts in food materials. Steps in image processing are image acquisition, Preprocessing of image, image segmentation, feature extraction and classification. In proposed framework they compare different types of filters and after comparison hybrid median filter was selected as the filter with the high PSNR value and is used in preprocessing. Two image segmentation techniques such as Colour based binary Image segmentation, Particle swarm optimization are compared and finally got that for food quality evaluation colour based binary image segmentation is well preformed.

Hamirul Aini Hambali, Sharifah Lailee Syed Abdullah, Nursuriati Jamil and Hazaruddin Harun et al [8]. This paper proposed a rule-based segmentation method to segment natural images correctly and accurately. To segment the images of interested object this method uses IF-THEN algorithm. Firstly input colored image in converted into RGB colour format and then Identify colour of investigated object if colour is black then apply adaptive thresholding otherwise apply adaptive k-means clustering method. Adaptive thresholding is work well for image with black colour objects. Otsu, K-means and Fuzzy C-means and proposed method, all four segmentation methods are implemented on fruit images and based on visual and quantitative evaluations their performances are compared.

Table 1 shows the comparative study ane analysis of all the related work.

\section{MORPHOLOGICAL OPERATIONS}

Morphological image processing is a collection of nonlinear operations related to the shape Morphological techniques uses an image with a small shape called a structuring element. At all possible locations in the image the structuring element is compared with the corresponding neighborhood of pixels. If structuring element fits into the neighborhood pixels then replace all neighborhood pixel values by 1 otherwise replace by 0 . The structuring element is a small binary image matrix each with a value of zero or one. There are some fundamental and compound morphological operations such as Erosion Dilation and Opening, Closing [9].

1. Erosion removes small details from a binary image and reduces the size of regions of interest. From both the inner and outer boundaries of regions structuring elements shrinks an image. The holes and gaps between different regions become larger.

2. Dilation has the opposite effect to erosion it adds a layer of pixels to both the inner and outer boundaries of regions. The holes and gaps between different regions become smaller.

3. Opening of an image by a structuring element is erosion followed by dilation. Opening can open up a gap between objects connected by a thin bridge of pixels.

4. Closing of an image by a structuring element is a dilation followed by erosion. Closing fill holes in the regions while keeping the initial region sizes.

\section{PROPOSED WORK}

The Proposed work is focus on segmenting the small overlapped regions from the Input food image. The different image segmentation techniques based on edge detection, region growing, based on clustering and thresholding are applied on food images but these techniques are not useful for segmenting the overlapped regions of food. Watershed segmentation technique is used to solve the problem of over segmentation and segmenting small regions which are overlapped to each other but this segmentation results are not more accurate for increasing accuracy of segmentation result. 
Hybrid model is proposed by combining Morphological Operations and Watershed Segmentation technique (MOWS) to segment overlapped regions of food with their shape as shown in figure 2 .

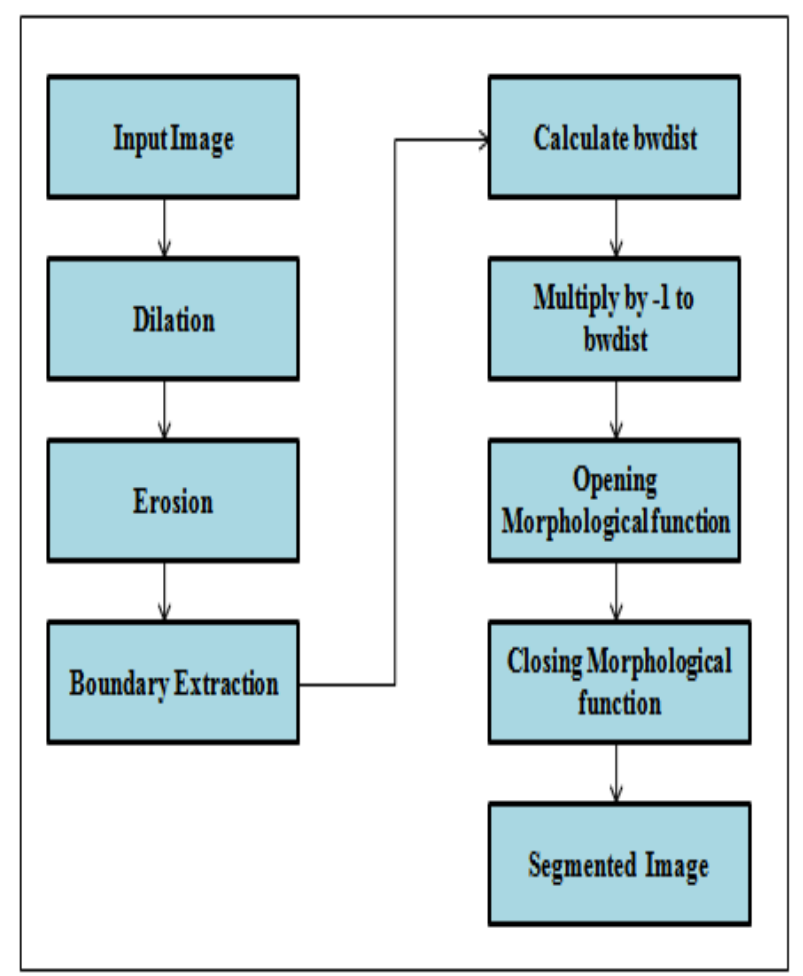

Figure 2: Proposed Work

The proposed model work as follows:

Step 1: Input Image is taken.

Step 2: The input image is dilated by structuring element.

Step 3: The input image is eroded by structuring element.

Step 4: Boundary extraction is done by subtracting eroded image from dilated image.

Step 5: Then apply Watershed segmentation algorithm.

Final output is segmented image

\section{EXPERIMENTAL RESULTS AND DISCUSSIONS}

To study and analyze Watershed image segmentation technique, food input images are taken from web. Basically input images having different fruits and all input images having different background and size. For experiment the image size taken as $200 * 200$ and 9 different images are taken as input. Figure 3 and figure 4 shows experimental result of Watershed image segmentation and Proposed Segmentation algorithm. Segmentation results are discussed on the basis of visual evaluations and quantitative. In Watershed Segmentation the regions are segmented as small regions, all thin edges are also detected but the original shape of the region is not getting properly. In Proposed algorithm the morphological operations such as dilation and erosion operations are used to extract shape of different regions but it is not accurately detect all boundary edges and some regions are not get closed, so after that watershed segmentation algorithm as applied and thin edges are also get detected. PSNR is used to calculate the peak signal-to-noise ratio.
PSNR is used to measure the quality of the image. PSNR values are calculated between original image and segmented image. Higher the PSNR value Quality of the Output image is good. Table 2 shows the performance comparison between Watershed Segmentation and Proposed MOWS algorithm using PSNR values. The PSNR Values of the MOWS are greater than the Watershed segmentation.

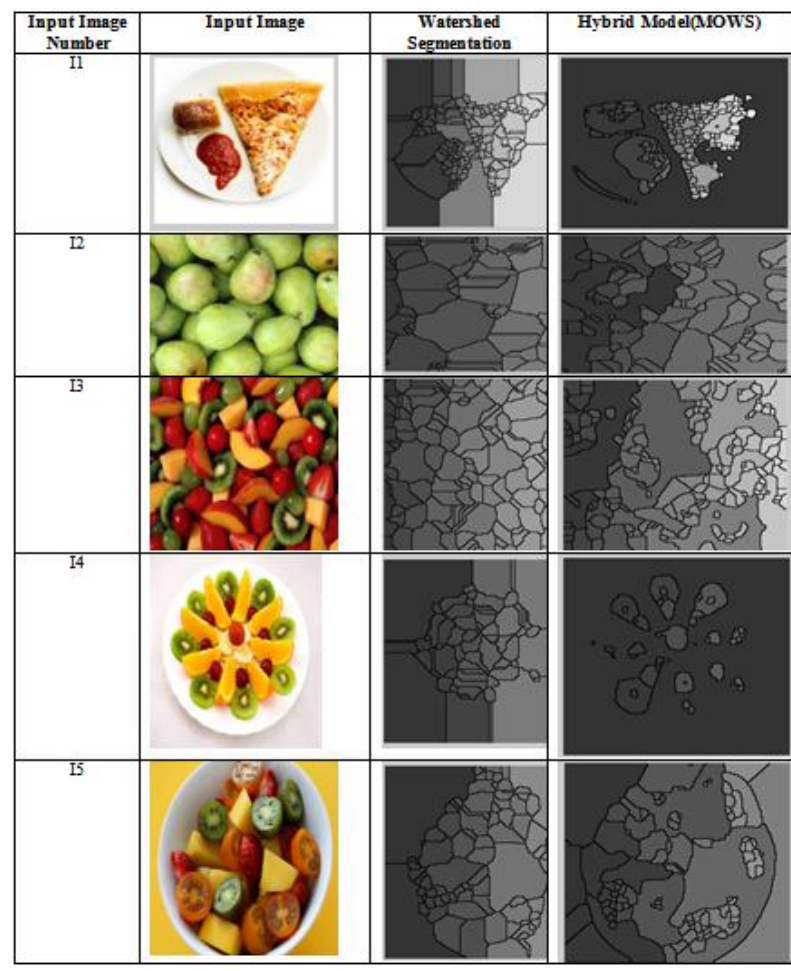

Figure 3: Output of Watershed Image Segmentation and Proposed MOWS from Input image I1-I5

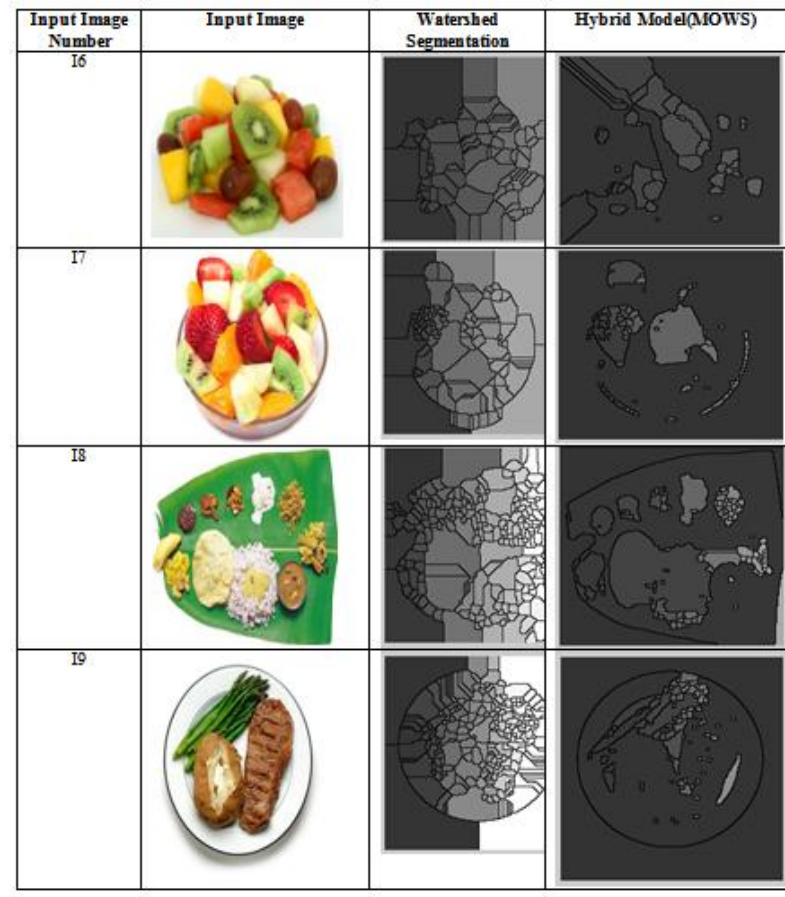

Figure 4: Output of Watershed Image Segmentation and Proposed MOWS from Input image I6-I9 


\section{CONCLUSION AND FUTURE SCOPE}

In this work new Image Segmentation method is proposed based on Morphological Operations and Watershed Segmentation (MOWS) whose goal is to overcome the problem of Watershed Segmentation. Watershed segmentation on the food image segmented the image into small regions, all thin edges are also detected but the original shape of the region is not getting properly. The proposed method is applied for segmenting the image into different regions with their shape. The morphological operations are applied on the input image before applying the watershed segmentation. By applying morphological Operations with watershed technique gives better results. As the boundary extraction is done by using morphological operations and then watershed technique is applied. From the comparison of PSNR values between the
Watershed segmentation and Proposed MOWS, it shows that the quality of the output image from Proposed MOWS is good than the watershed segmentation. In proposed method the morphological operations are used to detect all thin edges and getting the shape but it is not detecting the edges which are in diagonal directions so detecting the diagonal directions also in future combinations of morphological operations and gradient magnitude can be use.

Table 1: Comparative Analysis

\begin{tabular}{|c|c|c|c|c|}
\hline $\begin{array}{l}\text { Sr. } \\
\text { No }\end{array}$ & Paper Title & $\begin{array}{l}\text { Techniques } \\
\text { used }\end{array}$ & Input Image & Analysis \\
\hline 1 & $\begin{array}{l}\text { Watershed Image Segmentation Algorithm } \\
\text { Base on Particle Swarm and Region } \\
\text { Growing.(2015 IEEE) }\end{array}$ & $\begin{array}{l}\text { Watershed } \\
\text { algorithm, } \\
\text { region growing }\end{array}$ & All type of images & Solve problem of Over Segmentation. \\
\hline 2 & $\begin{array}{l}\text { An Improved Image Segmentation } \\
\text { Algorithm Based on the Watershed } \\
\text { Transform. (2014 IEEE) }\end{array}$ & $\begin{array}{l}\text { Watershed } \\
\text { algorithm, } \\
\text { marker } \\
\text { extraction. }\end{array}$ & Food image & $\begin{array}{l}\text { By modified gradient image reduce over } \\
\text { segmentation. }\end{array}$ \\
\hline 3 & $\begin{array}{l}\text { Segmentation of Color Image using } \\
\text { Adaptive Thresholding and Masking with } \\
\text { Watershed Algorithm. (2013 IEEE) }\end{array}$ & $\begin{array}{l}\text { Watershed } \\
\text { algorithm, } \\
\text { region growing }\end{array}$ & All type of images & $\begin{array}{l}\text { Adaptive thresholding and masking } \\
\text { reduce over segmentation. }\end{array}$ \\
\hline 4 & $\begin{array}{l}\text { Measuring Calorie and Nutrition From } \\
\text { Food Image.(2014 IEEE) }\end{array}$ & Edge Detection & $\begin{array}{l}\text { All type of food } \\
\text { images }\end{array}$ & $\begin{array}{l}\text { Unable to segment mixed food portion or } \\
\text { overlap portions }\end{array}$ \\
\hline 5 & $\begin{array}{l}\text { Image Segmentation for Food } \text { Quality } \\
\text { Evaluation Using Computer Vision } \\
\text { System.(2014 IJERA) }\end{array}$ & $\begin{array}{l}\text { Color based } \\
\text { Binary Image } \\
\text { and Particle- } \\
\text { Swarm } \\
\text { Optimization }\end{array}$ & Food images & $\begin{array}{l}\text { It is used to find defects in food } \\
\text { materials. }\end{array}$ \\
\hline 6 & $\begin{array}{l}\text { A Rule-based Segmentation Method for } \\
\text { Fruit Images Under Natural Illumination. } \\
\text { (2014 ICC) }\end{array}$ & $\begin{array}{l}\text { Otsu, K-mean, } \\
\text { Fuzzy } \quad \text { C- } \\
\text { means, If-Then }\end{array}$ & $\begin{array}{l}\text { Image containing } \\
\text { single food item }\end{array}$ & $\begin{array}{l}\text { It is not well suited for image containing } \\
\text { multiple food items. }\end{array}$ \\
\hline
\end{tabular}

Table 2 : Performance of Watershed Segmentation and Proposed MOWS algorithm using PSNR values.

\begin{tabular}{|c|c|c|}
\hline \multirow{2}{*}{$\begin{array}{c}\text { Input Image } \\
\text { Number }\end{array}$} & $\begin{array}{c}|c| \\
\text { Watershed } \\
\text { Segmentation }\end{array}$ & MOWS \\
\cline { 2 - 3 } & 112.24 & 112.28 \\
\hline I1 & 114.08 & 114.21 \\
\hline I2 & 126.18 & 126.16 \\
\hline I3 & 112.01 & 112.06 \\
\hline
\end{tabular}




\begin{tabular}{|c|c|c|}
\hline I5 & 116.40 & 116.60 \\
\hline I6 & 114.20 & 114.20 \\
\hline I7 & 112.26 & 112.40 \\
\hline I8 & 115.90 & 115.96 \\
\hline I9 & 113.34 & 113.60 \\
\hline
\end{tabular}

\section{REFERENCES}

[1] Y. He, N. Khanna, C.J. Boushey and E.J. Delp, Image Segmentation for Image-Based Dietary Assessment: A Comparative Study. 2013 IEEE.

[2] Rajeshwar Dass, Priyanka, Swapna Devi, "Image Segmentation Techniques", IJECT Vol. 3, Issue 1, Jan. March 2012.

[3] SUN Hui-jie, Watershed Image Segmentation Algorithm Base on Particle Swarm and Region Growing, 2015 IEEE.

[4] Xuemei Cui, Guowei Yang, Yan Deng, Shaolong Wu, An Improved Image Segmentation Algorithm Based on The Watershed Transform, 2014 IEEE.

[5] Md.Habibur Rahman and Md. Rafiqul Islam, Segmentation of Color Image using Adaptive Thresholding and Masking with Watershed Algorithm, 2013 IEEE
[6] Parisa Pouladzadeh, Shervin Shirmohammadi and Rana Al-Maghrabi, Measuring Calorie and Nutrition From Food Image. IEEE Transactions On Instrumentation And Measurement, Vol. 63, No. 8, August 2014.

[7] Nandhini. P and Dr. J. Jaya, Image Segmentation for Food Quality Evaluation Using Computer Vision System. Int. Journal of Engineering Research and Applications www.ijera.com ISSN : 2248-9622, Vol. 4, Issue 2( Version 5), February 2014.

[8] Hamirul Aini Hambali, Sharifah Lailee Syed Abdullah, Nursuriati Jamil and Hazaruddin Harun, A Rule-based Segmentation Method for Fruit Images under Natural Illumination. International Conference on Computer, Control, Informatics and Its Applications 2014.

[9] https://www.cs.auckland.ac.nz/courses/compsci773s1c/le ctures/ImageProcessinghtml 